\title{
Vagus Nerve Stimulation and the Cardiovascular System
}

\author{
Michael J. Capilupi, ${ }^{1}$ Samantha M. Kerath, ${ }^{2}$ and Lance B. Becker ${ }^{1,2,3}$ \\ ${ }^{1}$ Department of Emergency Medicine, North Shore University Hospital, Northwell Health, Manhasset, \\ New York 11030 \\ ${ }^{2}$ Feinstein Institute for Medical Research, Northwell Health, Manhasset, New York 11030 \\ ${ }^{3}$ Donald and Barbara Zucker School of Medicine at Hofstra/Northwell, Hempstead, New York 11549 \\ Correspondence: lance.becker@northwell.edu
}

The vagus nerve plays an important role in maintaining physiological homeostasis, which includes reflex pathways that regulate cardiac function. The link between vagus nerve activity and the high-frequency component of heart rate variability (HRV) has been well established, correlating with vagal tone. Recently, vagus nerve stimulation (VNS) has been investigated as a therapeutic for a multitude of diseases, such as treatment-resistant epilepsy, rheumatoid arthritis, Crohn's disease, and asthma. Because of the vagus nerve's innervation of the heart, VNS has been identified as a potential therapy for cardiovascular disorders, such as cardiac arrest, acute myocardial infarction, and stroke. Here, we review the current state of preclinical and clinical studies, as well as the potential application of VNS in relation to the cardiovascular system.

$\mathrm{T}_{\mathrm{p}}^{\mathrm{h}}$ he motor vagus nerve constitutes the main peripheral neural structure of the parasympathetic autonomic nervous system (ANS). The vagus contains efferent (motor) fibers that innervate smooth muscle cells of internal organs and vessels and endocrine and exocrine cells in the lung, the heart, the gastrointestinal tract, the pancreas, the liver, among others (Fig. 1). Among all those organs, the heart is innervated by the vagus at both cardiac muscle cells and at the conduction system. In particular, the atria, the sinoatrial node (SAN), the atrioventricular node (AVN), the ventricular myocardium, and the ventricular conduction system are all innervated by postganglionic efferent vagal fibers (Fig. 2). The effects of vagus nerve stimulation (VNS) can to some extent predicted from this anatomical distribution and they include: reduction in heart rate (negative chronotropic action on SAN), reduction in atrioventricular conduction (negative dromotropic action on AVN), changes in threshold for induction of atrial fibrillation (AF) (action on atrial conduction system), reduction in ventricular contractility (negative inotropic action on the ventricular myocardium), reduction in threshold for induction of ventricular arrhythmias (action on ventricular conduction system), etc. This article will review the experimental and clinical evidence for physiologic effects of VNS on the heart and their clinical significance.

Editors: Valentin A. Pavlov and Kevin J. Tracey

Additional Perspectives on Bioelectronic Medicine available at www.perspectivesinmedicine.org

Copyright (C) 2020 Cold Spring Harbor Laboratory Press; all rights reserved; doi: 10.1101/cshperspect.a034173

Cite this article as Cold Spring Harb Perspect Med 2020;10:a034173 
M.J. Capilupi et al.

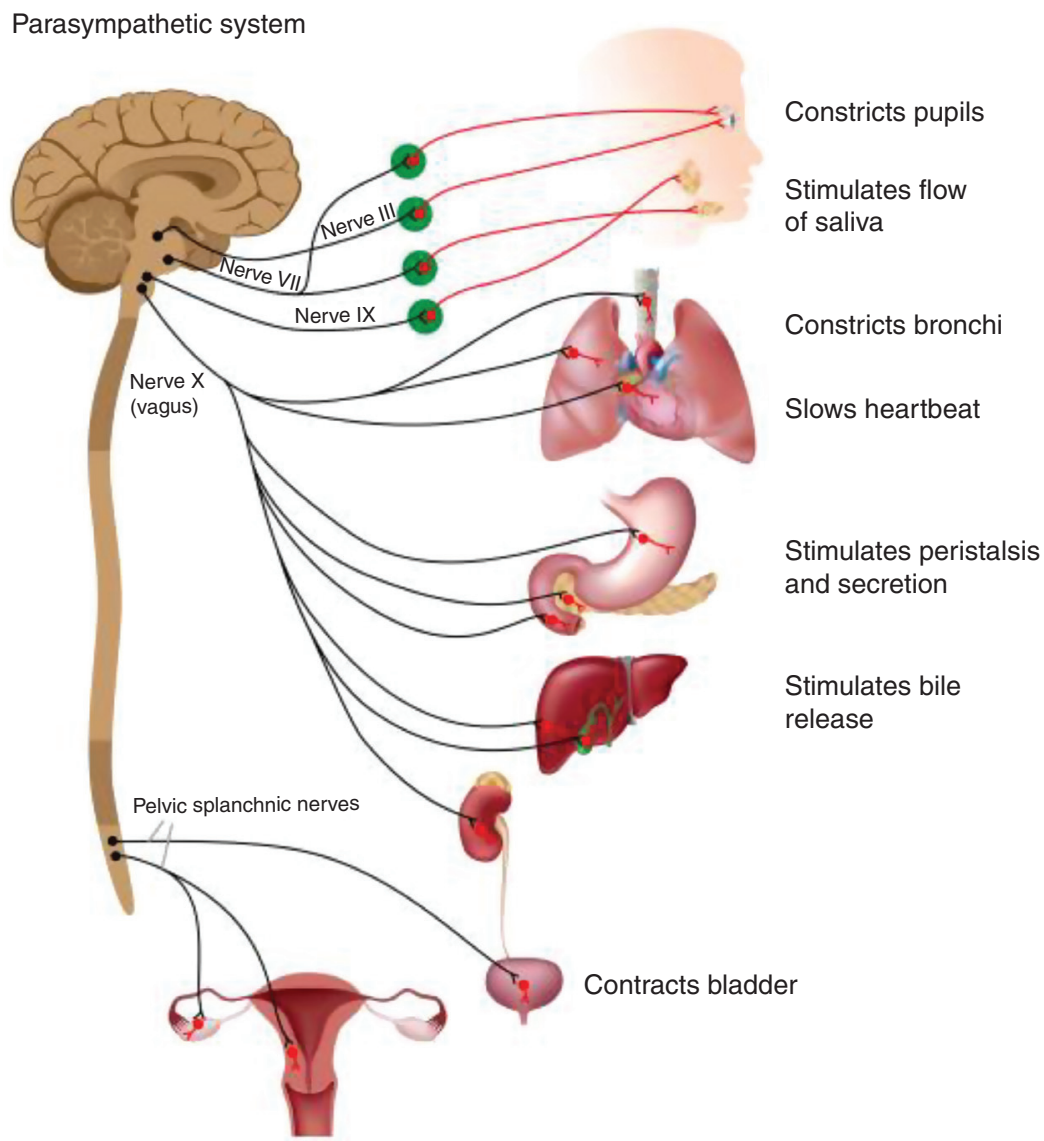

Figure 1. Schematic representation of the efferent (motor) projections of the parasympathetic autonomic nervous system (ANS). Cranial nerves III, VII, and IX innervate the pupils, producing pupillary constriction, and the salivary glands, resulting in increase in the flow of saliva. The pelvic splachnic nerves innervate the bladder, with their activation resulting in bladder constriction and facilitation of urination, the lower intestine, facilitating defecation; they also innervate the male and female sexual organs. The main efferent conduit of the parasympathetic ANS, the motor vagus, starts in cells in two nuclei in the brainstem, the dorsal motor nucleus of the vagus, and the nucleus ambiguus. The axons of those cells project to a variety of organs, forming synapses with ganglionic cells in parasympathetic ganglia found at or very close to those organs. Postganglionic axons from ganglionic cells innervate the lungs (promoting bronchoconstriction), the heart (slowing of heart rate, reduction in cardiac contractility), the pulmonary and systemic vessels (vasodilation and reduction in blood pressure), stomach (promoting peristalsis and gastric secretion), liver and pancreas (complex metabolic actions), spleen (preganglionic axons interacting with the splenic nerve, promoting anti-inflammatory effects), and kidneys (affecting vascular tone and filtration rate).

VNS was first developed in the late 19th century by American neurologist James Corning for the treatment of epilepsy (Yuan and Silberstein 2016a). Corning believed that epilepsy was a result of excessive cerebral blood flow, and hypothesized that VNS would reduce the increased blood flow (Yuan and Silberstein 2016a). While his initial attempt at using crude transcutaneous electrodes was unsuccessful, James Corning brought the concept of VNS into modern medicine (Yuan and Silberstein 2016a). In 1934, Soma Weiss discovered that compression of the carotid sinus produced syncope in humans, which differed from the same stimulation's effects on heart rate or blood pressure (Ferris et al. 1935). Weiss believed this 


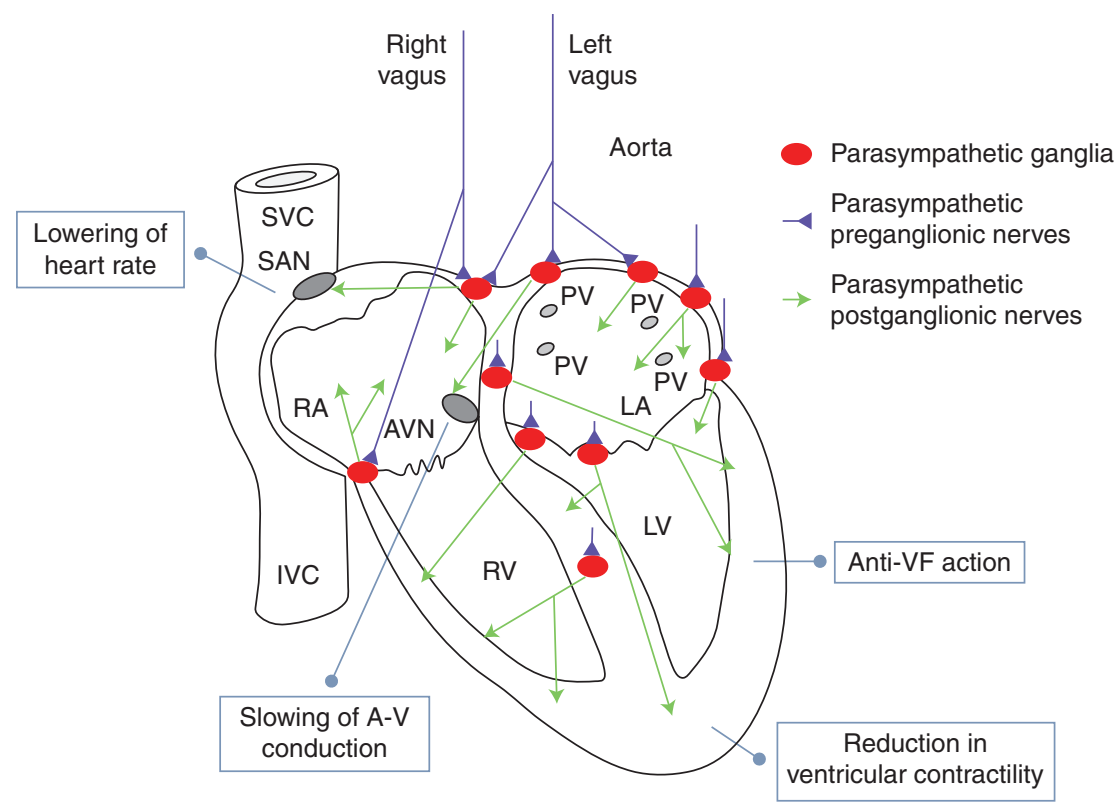

Figure 2. Vagus nerve innervations in the heart. Shown in blue are the two cardiac vagal preganglionic nerves (left and right). They synapse on a number of parasympathetic ganglia, shown in red, located in the epicardium and in the atrial and ventricular septum. The parasympathetic ganglionic neurons project postganglionic axons, shown in green, to different parts of the heart; the anatomical distribution of those axons can help explain the physiological actions of the vagus in the heart. In more detail: sinoatrial node (SAN): vagus nerve activation resulting in lowering of heart rate (negative chronotropic action); atrioventricular node (AVN): slowing of A-V conduction (negative dromotropic action); walls of the right and left ventricles (RVs, LVs) and conduction system: reduction in ventricular contractility (negative inotropic action); increase in threshold for induction of ventricular fibrillation (VF). SVC, Superior vena cava; IVC, inferior vena cava; RA/LA, right and left atrium; PV, pulmonary vein. (Image modified from Coote 2013, with permission, from The Physiological Society @ 2013.)

compression caused a direct cerebral response, as anatomically the carotid artery runs along the cervical vagus nerve, leading directly into the carotid sinus. Several years later, a study by Bailey and Bremer found that electroencephalogram changes occurred after the application of VNS (Bailey and Bremer 1938). In 1951, Dell and Olson found that stimulation of the cut vagus nerve caused responses in the thalamus (Dell and Olson 1951). The first successful experimental use of VNS in an animal model occurred in 1952 when it was shown to have an antiepileptic effect (Yuan and Silberstein 2016a) in cats with strychnine-induced seizures (Yuan and Silberstein 2016a). In 1985, researchers found that stimulation of the vagus nerve led to an alteration in electrical activity in the brain, which could terminate seizures in a canine model (Zabara 1985). The parameters for VNS can be tailored for optimum efficacy for the particular disease, application method, and individual patient.

While there was a heavy focus on the neurological effects of VNS, researchers at the time were also beginning to study the effect of VNS on cardiac function. In 1960, Sarnoff et al. found that VNS led to bradycardia and decreased contractility of the atria in canines. This decrease in atrial contractility led to less ventricular filling and stoke volume in their subjects meaning that the heart was less effective as a pump (Sarnoff et al. 1960). In addition to studying the effect of VNS on hemodynamics, scientists also examined the effect of VNS on heart rhythm. In 1975, Kolman et al. found that VNS had a protective effect against ventricular fibrillation (VF) in anesthetized canines (Kolman et al. 1975). Similar effects were found in a contemporane- 
M.J. Capilupi et al.

ous study that showed that VNS attenuates the positive ionotropic response seen during the administration of norepinephrine, from which it was concluded that there must be muscarinic acetylcholine receptors within the walls of the ventricles (Levy and Blattberg 1976).

Given the promising data from animal models, researchers began to assess the feasibility of using VNS in human subjects with neurological conditions. While the first vagus nerve stimulator in humans was implanted by Penry et al. in 1988, it was not until 1994 that VNS was shown to have antiepileptic properties in a human clinical trial (Penry and Dean 1990; Yuan and Silberstein 2016a). VNS treatment was shown to reduce seizure frequency after 14 weeks in patients suffering from chronic partial seizures (Yuan and Silberstein 2016a). Shortly thereafter, a VNS consortium carried out two pilot studies with programmable implantable devices, and found a $47 \%$ reduction in seizure frequency at 35-month follow-up (Ben-Menachem et al. 1994). Based on this work, the Food and Drug Administration (FDA) approved VNS as a treatment for refractory epilepsy in 1997 and depression in 2005 (Schacter and Schmidt 2000; Bonaz et al. 2016b).

While treatment with VNS has been attempted as early as the late 19th century, the mechanism behind its effects was poorly understood. Major growth in the field began in the early 2000s, with the indication that VNS could attenuate the inflammatory response induced by endotoxin and the identification of the inflammatory reflex (Borovikova et al. 2000; Tracey 2002). Stimulation of the vagus nerve was found to modulate the inflammatory reflex, and later studies identified the $\alpha 7$ nicotinic acetylcholine receptor $(\alpha 7 \mathrm{nAchR})$ as a critical target for the attenuation of proinflammatory cytokine release (Parrish et al. 2008; Olofsson et al. 2012; Pavlov et al. 2018). The anti-tumor necrosis factor $\alpha(\mathrm{TNF}-\alpha)$ property of the cholinergic antiinflammatory pathway also indicates VNS as a treatment in therapeutic targets, such as inflammatory bowel disease (IBD) and rheumatoid arthritis (RA), which are driven by TNF- $\alpha$ (Meregnani et al. 2011; Bonaz and Bernstein 2013; Bonaz et al. 2016a,b; Koopman et al. 2016).
Given its proven impact on the inflammatory response in animal models, VNS is currently undergoing multiple trials to determine its efficacy in various diseases, such as treatment-resistant epilepsy, autoimmune disorders, heart failure, and asthma (Yuan and Silberstein 2016b). Other diseases, such as cardiovascular disease (CVD), are presenting promising preclinical data, and may be candidates for future clinical trials in humans. Recently, the field of research has expanded to examine the use of VNS as a treatment modality in several CVDs, such as cardiac arrest and stroke. Our aim is to review the current state of research into the use of VNS, as well as its applications related to the cardiovascular system

\section{CARDIAC ARREST}

Cardiac arrest is the complete cessation of blood flow from the heart. Typically, this occurs rapidly as a result of a malignant cardiac arrhythmia such as VF. If untreated, it uniformly results in the complete loss of heartbeat and rapidly results in death. However, it can be treated effectively with rapid initiation of high-quality cardiopulmonary resuscitation (CPR) with chest compressions, defibrillation, ventilation, drugs, and other advanced resuscitation techniques; however, all of these interventions are highly timesensitive. The chances of death significantly increase within the first few minutes without treatment. The current paradigm for the treatment of cardiac arrest is described by the "threephase model," where the first 5 min are denoted as the electrical phase wherein defibrillation is highly effective (Weisfeldt and Becker 2002). A second phase, the circulatory phase, begins after $\sim 5 \mathrm{~min}$ and last until $10 \mathrm{~min}$, and can be effectively treated by high-quality CPR followed by defibrillation. The third, or metabolic phase, occurs after a mere $10 \mathrm{~min}$ and is highly lethal; current advanced cardiac life support (ACLS) is not very effective in this phase and the vast majority of victims of cardiac arrest die within this phase. The three-phase model highlights the fact that the treatment of cardiac arrest is very time-sensitive and that different therapies are considered at different timepoints after initia- 
tion of cardiac arrest. In this model, the treatment of cardiac arrest is not a "one-size-fits-all" disease, and the future treatments of cardiac arrest must begin to take this into account. Cardiac arrest is a leading cause of death in the United States, with an out-of-hospital cardiac arrest (OCHA) incidence of $\sim 356,461$, an in-hospital cardiac arrest (IHCA) incidence of $\sim 209,000$, and a nearly $82 \%$ fatality rate (Benjamin et al. 2018). Given the high mortality rate, and the long-term morbidity for survivors, research focusing on developing alternative treatment for cardiac arrest in animal models has become a priority.

Preclinical studies have been undertaken to assess whether VNS could be used to facilitate resuscitation attempts in rats with induced cardiac arrest (Sun et al. 2018). It was found that when VNS was used in conjunction with CPR in rodents, success rates for return of spontaneous circulation (ROSC) were $90.91 \%$ when compared to CPR alone at $83.33 \%$ (Sun et al. 2018). Results also indicated that VNS reduced the duration of CPR by preventing arrhythmias independent of the heart slowing mechanism expected in VNS (Sun et al. 2018). In addition to its effect on CPR, VNS also reduced the number of electrical shocks required to achieve ROSC (Sun et al. 2018). This may be related to the fact that increased sympathetic activity could be responsible for failed defibrillation. Applying VNS during resuscitation attempts would dampen the effects of the sympathetic ANS on the heart thus improving the efficacy of defibrillation. Experimental animals that received VNS also showed lower rates of myocardial ischemia after CPR than those that did not, which was attributed to the decreased use of oxygen in the heart because of a lower heart rate. Finally, the mice that received VNS treatment had an increased 72 -h survival rate than those that did not receive VNS (Sun et al. 2018). Researchers postulated that the protective effects of VNS may be related to $\alpha 7 \mathrm{nAChR}$, and the anti-inflammatory effects of VNS. These effects have been shown in several studies that elucidated the effects of the inflammatory reflex.

In addition to its ability to aid in recovery from cardiac arrest, VNS has also been shown to directly affect ventricular function; this is particularly important in light of the fact that ventricular arrhythmias, such as VF, are responsible for a large portion of sudden cardiac deaths (AlKhatib and Stevenson 2018; Camm et al. 2018). In 2014, researchers found that VNS directly modulated left ventricular function in pigs and sheep (Naggar et al. 2014). This was believed to be the result of VNS affecting the spread of the dominant frequency during VF. The dominant frequency is the waveform that is largest in amplitude and most directly related to the rate of contraction of the ventricles. VNS was also shown to increase both action potential duration (APD) and the effective refractory period (ERP), which decreases intracellular calcium. In turn, this decreases contractility therefore decreasing left ventricular wall motion (Naggar et al. 2014). Additionally, when VNS was applied, there was increased electrical heterogeneity in ventricular myocytes. While VNS has a demonstrable positive impact on the heart following cardiac arrest, the authors found that VNS can also lead to a significant decrease in blood pressure, which can potentially induce ischemia in organs ( Naggar et al. 2014).

Ventricular arrhythmia has also been found to be related to a reduction in vagal tone $(\mathrm{Ng}$ 2014; Lee et al. 2018a; Winter et al. 2018). This effect has been studied on isolated innervated rabbit hearts while increasing sympathetic and parasympathetic tone (Winter et al. 2018). They found that increased sympathetic tone was proarrhythmic in long QT-associated arrhythmias, with VNS attenuating these effects (Winter et al. 2018). When isolated, nondenervated hearts were studied, rich vagal nerve innervation to the ventricles was found, which could be exploited to help protect against VF (Ng 2014). This protection could be a result of the fact that VNS releases nitrous oxide $\left(\mathrm{N}_{2} \mathrm{O}\right)$ via an independent nitrergic neural network, which regulates cardiac physiology ( $\mathrm{Ng} 2014)$. To explore this possibility, the efficacy of VNS was tested in the presence of a muscarinic block, and it was found that VNS was still able to reduce the incidence of VF ( $\mathrm{Ng} 2014)$. Similar effects were found in rabbit hearts treated with a nitric oxide (NO) synthase inhibitor before VNS. In 
M.J. Capilupi et al.

the presence of the inhibitor, the VF threshold and ERP were not affected; the antifibrillatory effects of VNS were restored with the removal of the inhibitory blockade (Brack et al. 2007). Others have also found that reduced vagal tone is a contributor in the development of VF, and that VNS acts on heart rate and heart rhythm via $G$ protein-gated inwardly rectifying $\mathrm{K}^{+}$(GIRK) channels (Lee et al. 2018a). When applying VNS to a transgenic GIRK channel knockout (KO) mouse model, no significant response was observed, indicating that GIRK is necessary for the effects of VNS (Lee et al. 2018a). These findings were recently corroborated in a study that indicates that activation of GIRK is primarily responsible for the changes in heart rate dynamics (i.e., heart rate variability [HRV]) seen in VNS (Lee et al. 2018b). These results are promising, and the connection between NO, GIRK, and VF should be investigated further in both preclinical models and clinical trials.

While VNS has been shown to aid in recovery from cardiac arrest, there is no doubt that it must be used carefully, as it has also been shown to be effective in inducing asystole for use in novel surgical techniques (Ronson et al. 2003). Research has shown that pharmacologically potentiated VNS can be used to induce controlled intermittent asystole in a canine model, creating an "on-off" switch that can be used to perform minimally invasive heart surgery in the future. By stimulating the vagus nerve for a cycle of 12 sec "on" and $15 \mathrm{sec}$ "off," 15 times in each dog, researchers were able to effectively create an "on-off" switch, which also had a low incidence of escape beats. Furthermore, they showed that repetitive periods of transient asystole showed no evidence of myocardial or neurological injury (Ronson et al. 2003). Despite the decrease in coronary perfusion during these periods, they postulated that a reduction in neutrophil accumulation may have been the mechanism that allowed them to avoid injuring the myocardium.

Overall, the results from these studies show that VNS has a future role in the treatment of cardiac arrest, as well as for use in novel surgical techniques. The precise mechanism by which VNS serves as a cardioprotective strategy remains an important area of research. While these have only been tested in animal models, the fact that VNS has been shown to have similar effects across several species implies that similar results may be possible in humans.

\section{HEART FAILURE}

Heart failure is a condition in which the heart muscle is unable to maintain sufficient levels of blood flow to meet the body's needs. The causes of heart failure include a weaker-than-normal heart muscle, abnormalities of the heart valves, infection, coronary artery disease, and many other possible etiologies. The decreased ability to pump blood results in decrease blood flow to the kidneys, which can result in water retention; this is termed congestive heart failure (CHF). $\mathrm{CHF}$ is considered to be a progressive disease although with treatment patients may live for an extended period of time. The heart will typically attempt to adapt to CHF by increasing in mass, and by beating faster, but these adaptive mechanisms will eventually fail to keep up with the demand for blood flow. Patients become progressively more symptomatic, and many people die from CFH despite treatment (Savarese and Lund 2017). There are currently nearly 5.7 million Americans with CHF, and >900,000 new diagnosis of $\mathrm{CFH}$ occur each year. The worldwide healthcare costs of $\mathrm{CHF}$ are roughly $10 \%$ of all cardiovascular costs, which amounts to $\$ 31$ billion dollars (in 2012) and is rising rapidly with the aging of the population.

VNS is an interesting new modality to consider in relation to heart failure. Relevant to VNS modulation of the parasympathetic nervous system, researchers postulated that an abnormal imbalance between the sympathetic and parasympathetic nervous systems exists during heart failure, in which the sympathetic nervous system becomes overactive and worsens heart failure (Kishi 2012). The hyperactivity of the sympathetic nervous system causes afferent sympathetic signaling, which leads to tonic and reflex inhibition of cardiac vagal efferent activity; sympathetic nervous system hyperactivity can also contribute to left ventricular diastolic dysfunction and to an increased cardiovascular risk. VNS by itself, as well as alongside $\beta$-blockers, 
has been shown to produce significant improvement in left ventricular hemodynamics in animals with heart failure and to decrease mortality from $50 \%$ to $14 \%$ (Kishi 2012).

Similar cardioprotective results with VNS have been found in canine models of intracoronary microembolization-induced heart failure (Hamann et al. 2013). It has been theorized that low-level VNS (LL-VNS) has a positive impact on sympathetic drive, the reduction of proinflammatory cytokines, NO elaboration, and the myocardial expression of gap junction proteins to which improvement in heart failure could be attributed (Hamann et al. 2013). It was also found that in various animal models, VNS suppressed ventricular arrhythmias, ventricular remodeling, and sudden cardiac death, in addition to the improvement in left ventricular function found by Kishi (Sabbah et al. 2011). A reduction in heart rate, attenuation of sympathetic overdrive, and down-regulation of the reninangiotensin-aldosterone system likely play a key role in this reduction in dysfunctional cardiac activity (Sabbah et al. 2011). Several studies have previously shown that heart failure patients have significantly elevated levels of inflammatory markers such as TNF- $\alpha$ and interleukin (IL)-6, which can predict a greater severity of coronary symptoms and a reduced response to treatments, such as cardiac resynchronization therapy (CRT) (Levine et al. 1990; Nozaki et al. 1997; Koller-Strametz et al. 1998; Tsutsumi et al. 2008; Hartupee and Mann 2013; Rordorf et al. 2014; Mann 2015). VNS was been shown to normalize expression of proinflammatory cytokines, such as TNF- $\alpha$ and IL-6, making it a promising therapy (Sabbah et al. 2011).

Researchers have also studied the effect of VNS on cardiac remodeling in heart failure (Sabbah et al. 2011; Hamann et al. 2013). One study found that VNS therapy improves left ventricular systolic function, prevents progressive left ventricular enlargement, and improves heart failure biomarkers when compared to controls. However, it was found that VNS must be applied chronically to be effective, in their study, for 6 months. When VNS treatment was terminated after 3 months, heart failure severity returned to baseline (Hamann et al. 2013). VNS has also been shown to have an impact on signaling pathways, including the baroreceptor reflex, suppression of proinflammatory cytokines, normalization of the NO pathway, and suppression of gap junction remodeling (Sabbah et al. 2011). VNS therapy was shown to improve left ventricular ejection fraction and decreased end-systolic and end-diastolic volumes. Additionally, VNS reduced preload improved left ventricular function without increasing oxygen consumption. Related to remodeling of the ventricles, VNS was shown to decrease the volume fraction of replacement fibrosis volume fraction of reactive interstitial fibrosis, myocyte cross-sectional area, and oxygen diffusion distance while also significantly increasing capillary density (Sabbah et al. 2011).

Recently, clinical trials have been performed examining the efficacy of VNS in human heart failure patients. The ANTHEM-HF trial used VNS in 59 heart failure patients, and found that VNS caused subtle changes in heart rate when applied on both the right and left side of the body. They concluded that the use of VNS in human subjects is a viable option for the modulation of the effects of the ANS on the heart (Nearing et al. 2016). Another large-scale trial is the INOVATE-HF trial, in which 707 patients were followed over several months. Those who received VNS treatment received an implantable BioControl CardioFit device. VNS was not found to improve the rate of death from any cause or heart failure events or reverse cardiac remodeling. VNS did, however, show significant improvements in 6-min hall walk duration, New York Heart Association (NYHA) functional class, and KCCQ quality of life (QoL) scores (Gold et al. 2016). Similar results were found in the NECTAR-HF trial of 96 heart failure patients given 6 months of chronic VNS therapy, which found no significant improvements in ventricular remodeling, function, or circulating cytokines; however, improvement was shown in NYHA functional class and other HF-related QoL scores (Zannad et al. 2015).

\section{ACUTE MYOCARDIAL INFARCTION}

Myocardial infarction (MI), commonly referred to as a "heart attack," occurs when the heart 
M.J. Capilupi et al.

becomes damaged because of a lack of perfusion, resulting from the occlusion of one or more coronary arteries. $\mathrm{MI}$ is the acute result of coronary artery disease or coronary heart disease. The prevalence of coronary heart and coronary artery disease in the United States is over 15 million people (Sanchis-Gomar et al. 2016). Nearly 120,000 American will die each year from MI, especially if the MI causes a sudden cardiac arrest (Benjamin et al. 2017). Not all patients with coronary artery disease will suffer an MI, but the presence of coronary artery disease is strongly associated with the development of MI. VNS is being investigated as the setting of MI with some studies evaluating the impact of VNS on blood flow (a principal driver of $\mathrm{MI}$ ), some examining the impact of VNS on arrhythmias, inflammatory changes, progression of heart disease, cardiac remodeling, and coronary heart disease progression.

VNS is an interesting modality for possible use in MI given its ability to modulate parasympathetic tone. The vagus nerve signals to the heart via the intracardiac ganglia, with the atria extensively innervated by parasympathetic vagal nerves (Sanchis-Gomar et al. 2016). It has been hypothesized that VNS might modulate atrial electrophysiological properties because of this innervation (Chen and Tan 2007). It has been shown in ambulatory canines that there is anatomical remodeling of stellate ganglia and that there is an increase in sympathetic activity relative to parasympathetic activity post-MI (Han et al. 2012). As discussed earlier, autonomic mismatch has been shown to increase the likelihood of arrhythmia, which was also found in this study (Han et al. 2012). The cardioprotective effects of VNS have also been shown in swine models, in which it was found that VNS provided protection as a result of increasing phosphorylation of connexin 43, which leads to a reduction in the time from $\mathrm{T}$-wave peak to end (Shinlapawittayatorn et al. 2014). This implies that VNS mainly improves ventricular repolarization, but not depolarization. Finally, the effect of VNS on post-MI remodeling of myocytes and the intrinsic cardiac neuronal system was studied in a guinea pig model (Beaumont et al. 2015). At 90-day post-MI, experimental animals were found to have an increased left ventricular end systolic volume and reduced ejection fraction compared to baseline; these effects were mitigated by VNS. It was also found that VNS induced enhanced glycogenolysis, which blunted the proapoptotic Bcl-2-associated X protein typically seen in MI (Beaumont et al. 2015).

LL-VNS has also been explored as a potential treatment option for MI as a combination therapy with other treatment options in swine, rat, and canine models. When applied during injury reperfusion, LL-VNS was shown to improve ventricular function and caused a dramatic reduction in ventricular tachycardia/VF episodes (Laurita and Hirose 2013). These protective properties could be attributed to the antagonization of sympathetic outflow to the heart, inhibition of $\beta$-adrenergic signaling, and the L-type calcium channel. In addition to this, LL-VNS may reduce reactive oxygen species and preserve myocardial connexin 43 . In addition to improving heart function, LL-VNS was also shown to decrease infarct size (Laurita and Hirose 2013). This was theorized to be a result of attenuation of reactive oxygen species and swelling and could be related to the nicotinic pathway within the inflammatory reflex.

Adding to this, transcutaneous LL-VNS was found to be efficacious in ameliorating post-MI remodeling in the myocardium (Wang et al. 2015). LL-VNS and its effect on calcium handling in rats post-MI has also been examined; LL-VNS improved left ventricular ejection fraction and attenuated myocardial interstitial fibrosis post-MI (Zhang et al. 2016). Plasma levels of norepinephrine and dopamine, but not epinephrine, were also reduced by LL-VNS. These effects were correlated with the restoration of myocardial SERCA2a, NCX1, and PLB during VNS. Subcellular levels of these calcium-handling proteins were decreased post-MI, and were shown to be restored when LL-VNS was applied (Zhang et al. 2016). This has implications for restoring heart function back to baseline after MI.

Studies have also shown the electrophysiological effect of VNS in relation to the electrical activity of the heart, in which intrinsic cardiac 
neurons showed depolarization of resting membrane potentials and increased input resistance, which were reduced in the VNS group (Beaumont et al. 2015). Neuronal excitability and sensitivity to norepinephrine remained increased in both VNS and non-VNS groups post-MI (Beaumont et al. 2015). It has also been found that MI decreases APD at both 50\% and $80 \%$ repolarization, and increases APD heterogeneity (Xie et al. 2014). Returning APD to its baseline values subsequently reduced the risk for arrhythmia after MI (Xie et al. 2014). Similar results were found in swine hearts, where MI increased sympathetic output to parasympathetic convergent neurons (Vaseghi et al. 2017). Application of VNS significantly reduced ventricular arrhythmia inducibility by decreasing ventricular excitability and heterogeneity of repolarization (Vaseghi et al. 2017).

VNS has also been shown to demonstrate antiapoptotic and anti-inflammatory effects on the myocardium (Han et al. 2012; Shinlapawittayatorn et al. 2014). This is due to a decrease in cytochrome $c$ release and an increase in IL-4 release. As a result, VNS was able to reduce the infarct size by $59 \%$ (Shinlapawittayatorn et al. 2014). It was found that timing of VNS was crucial and that VNS must be applied during ischemia and before the onset of reperfusion (Shinlapawittayatorn et al. 2014). Another study found that rats treated with VNS post-MI showed a significant reduction in TNF- $\alpha$ expression (Kong et al. 2012). Additionally, they found that MI decreased the baroreceptor reflex response and that VNS restored this to baseline. Finally, MI was shown to decrease the $\alpha 7 \mathrm{nAch} R$ levels and rats that received VNS had receptor levels that approximated baseline (Kong et al. 2012). These receptors have been previously implicated in the anti-inflammatory properties seen in VNS.

\section{ATRIAL FIBRILLATION}

$\mathrm{AF}$ is a relatively common cardiac arrhythmia characterized by rapid and irregular beating of the atria, which has been well established as a leading risk factor for stroke (Wolf et al. 1991; Gage et al. 2001; Heeringa et al. 2006; Schmitt et al. 2009). AF is associated with advancing age, as well as many other heart disease risk factors such as elevated blood pressure, diabetes, obesity, alcohol use, and many other factors (Staerk et al. 2017). Somewhere between 3 and 6 million Americans have AF (Mozaffarian et al. 2015), and it is responsible for more than 750 hospital admissions each year. The treatment of AF focuses on the control of heart rate and on anticoagulation for stroke prevention. In some patients, it is possible to restore a normal sinus rhythm (i.e., to stop the AF and restore a normal heartbeat). However, in most patients, this goal cannot be achieved, and many patients who have an AF episode go on to develop chronic AF.

While the precise cause of AF is not well understood, the cardiac factors related to sympathetic and parasympathetic tone are often believed to influence AF, thus the use of VNS in AF is undergoing ongoing research. It has been shown that the ANS plays an important role in the initiation and maintenance of AF; overactivation of the sympathetic ANS has been attributed to an increased risk of AF (Mozaffarian et al. 2015). Given its role in modulating the autonomic nervous response, LL-VNS has been shown to reduce the risk of AF in preclinical models (Sheng et al. 2011; Yu et al. 2012; Carpenter et al. 2015; Chen et al. 2015; Li et al. 2015; Mozaffarian et al. 2015).

Extensive work has been performed to assess the effects of LL-VNS on models assessing atrial remodeling and AF inducibility. Yu et al. examined the use of LL-VNS in the treatment of AF (Carpenter et al. 2015) in which they induced AF using rapid atrial pacing (RAP) in canines. Results from this study found that LL-VNS can reverse RAP-induced atrial remodeling and inhibit AF inducibility (Carpenter et al. 2015), while the group later established that electrical and autonomic remodeling work together to enhance the likelihood of AF (Yu et al. 2012). The study showed that LL-VNS was able to halt the remodeling of both the electrical and autonomic systems, and reduce the likelihood of AF (Yu et al. 2012). Similar studies have been performed in which AF was induced in a canine model using RAP, and treated with LL-VNS, finding that LL-VNS reversed remodeling of 
M.J. Capilupi et al.

the atria and reduced inducibility of AF (Yu et al. 2012). This effect may be attributed to stimulation of the cholinergic pathway, and inhibition of the intrinsic cardiac nervous system seen when LL-VNS was applied (Yu et al. 2012).

LL-VNS has also been shown to be effective in returning RAP-induced decreases in an ERP, increases in the window of vulnerability for AF inducibility, and restoration of neural activity to baseline values (Carpenter et al. 2015). These effects have been attributed to LL-VNS inhibition of the intrinsic cardiac ANS. In addition, these results have been achieved by delivering the LL-VNS through the auricular branch of the vagus nerve, showing that these effective treatments can be achieved noninvasively (Carpenter et al. 2015). Additionally, researchers have induced AF in the pulmonary vein and atrial appendage sites in a canine model to examine LL-VNS and its effect on focal AF. Results show that LL-VNS was able to prevent AF caused by rapid firing at both the pulmonary vein and nonpulmonary vein sites ( $\mathrm{Yu}$ et al. 2012). It was proposed that LL-VNS exerted its effects via the inhibition of neural activity of the ganglionated plexus (Yu et al. 2012), which has been previously shown to mediate the response to VNS (Jiang et al. 2018), possibly through Nav1.8 (Qi et al. 2014).

Studies have also been done to examine the effect of LL-VNS on gene expression on gap junctions in canines. After using RAP to induce $\mathrm{AF}$, it was observed that RAP could reduce the levels of atrial connexins $\mathrm{Cx} 40$ and $\mathrm{Cx} 43$, but LL-VNS was able to prevent the loss of the connexin expression (Li et al. 2015). Additionally, it was found that LL-VNS was able to suppress AF by shortening AF duration and prolonging the AF cycle length. They concluded that the antiAF properties of VNS could be attributed to the prevention of the loss of $\mathrm{Cx} 40$ and $\mathrm{Cx} 43$, which have previously been shown to reduce the incidence of ventricular tacchyarrythmias (Lerner et al. 2000).

\section{STROKE}

A stroke is a critical lack of perfusion to the brain that results in the death of an area of brain tissue.
Stroke represents a both a leading cause of mortality worldwide, as well as a large healthcare burden in the United States, costing $\sim \$ 40.1$ billion per year (Ovbiagele et al. 2013). With $\sim 795,000$ new or recurrent strokes each year, there remains a large unmet clinical need. Current projections indicate that the prevalence of stroke in the U.S. adult population will increase by $20.5 \%$ by 2030 over 2012 levels (Ovbiagele et al. 2013). Given the public health relevance, VNS has been examined as a possible tool to help treat stroke. Neuroinflammatory injury has been cited as a major cause of acute neuronal damage in stroke. Inflammatory cytokines and adhesion molecules recruit neutrophils, macrophages, and activate microglia. This recruitment leads to endothelial damage and the failure of the blood-brain barrier, which results in intracerebral hemorrhage and edema (Cai et al. 2014). The vagus nerve has been previously shown to play a role in the regulation of both peripheral and central inflammation (Heneka and O'Banion 2007). Given its role in regulation of inflammation, decreased vagus activity may predispose an individual to stroke, or the stroke itself may alter vagal inflammatory properties. In addition to this, the stroke itself may alter vagal immunoregulatory functions and may lead to the augmentation of inflammation in the periphery, as well as in the brain (Heneka and O'Banion 2007).

Numerous studies have shown the effect of VNS on the brain following ischemic stroke, particularly as it pertains to plasticity (Miller et al. 2009; Mravec 2010; Khodaparast et al. 2013; Meyers et al. 2018). One study showed that rats with unilateral ischemic lesions of the primary motor cortex doubled the recovery hit rate performance for forelimb strength when compared to training without VNS (Mravec 2010). This improvement was attributed to the recovery of enhanced plasticity seen within the motor cortex following VNS. This plasticity is likely a result of the release of acetylcholine, norepinephrine, and brain-derived neurotrophic factor; the necessity of the cholinergic nucleus basalis in the improvement of cortical plasticity has also been shown (Hulsey et al. 2016). A 2018 study used a retrograde transsynaptic trac- 
er, PRV-152, injected into the forelimbs of study rats, which had received both VNS and rehabilitation. A significant increase was found in PRVpositive cells in both the lesioned and unlesioned hemispheres of the brain. These findings indicate that the efferent motor circuits display significantly increased synaptic connectivity in animals who receive both VNS and rehabilitation (Meyers et al. 2018).

Prior results were replicated in a 2016 study, in which rats were implanted with cuff electrodes and stimulated 4 weeks poststroke (Khodaparast et al. 2016). Animals were found to have chronic forelimb function impairment, which was significantly attenuated by VNS paired with rehabilitative training, compared with rehabilitative training alone. This substantiated findings that rehabilitation alone was not a sufficient treatment, and that VNS had significant effect on poststroke limb movement recovery and forelimb strength and forelimb rotational function (Khodaparast et al. 2013, 2014). A series of similar experiments also showed the effectiveness of VNS in rehabilitative recovery following intracerebral hemorrhage (Hays et al. 2014a) as well as that VNS can help with poststroke recovery, even in elderly rats (Hays et al. 2016).

In addition to the significantly increased efficacy of stroke rehabilitation when paired with VNS, evidence is also emerging that there is a critical temporal component to this effect. Recent studies have shown that, to be effective, VNS must be paired temporally with rehabilitative training, as opposed to immediately after training, or with a delay after training (Hays et al. 2014b). This improved recovery persisted even past the cessation of VNS treatment, indicating that the benefits can be long-lasting. These results were echoed in a 2018 study, which found that, compared to a rehab only group, the VNS + rehab group had significant improvement on assessments of turn angle and success rate assessments at $7 \mathrm{wk}$ poststroke (Meyers et al. 2018).

VNS has also been shown to decrease lesion size and to attenuate cerebral edema after brain injury (Cai et al. 2014). Decreased activity of the inflammatory reflex has been shown to worsen neurological symptoms in ischemic stroke pa- tients, and researchers have shown that VNS reduced the incidence of cerebral edema caused by brain injury by reducing cerebral blood flow. VNS has also been shown to play a role in reducing stroke volume by as much as $50 \%$ by attenuating excitotoxicity and inhibiting inflammation, and thereby significantly improving clinical outcomes (Ay et al. 2011; Cai et al. 2014). This reduction could be related to decreased levels of glutamate after VNS treatment, as glutamate excitotoxicity has been found to play a role in stroke. Overall, the anti-inflammatory effects of VNS, increased GABA levels, and the activation of several genes involved in the suppression of inflammatory and apoptotic pathways play a critical role in the attenuation of stroke recovery (Ay et al. 2011; Cai et al. 2014).

While the effects of VNS paired with rehabilitation have been shown extensively in preclinical models, evidence is beginning to emerge supporting its efficacy in humans (Dawson et al. 2016; Capone et al. 2017; Kilgard et al. 2018; Kimberley et al. 2018). A small pilot study was conducted in Italy in 2016 to assess the safety and feasibility of transcutaneous VNS (tVNS) in conjunction with robotically assisted rehabilitation in patients with either chronic ischemic or hemorrhagic stroke. The tVNS group showed significantly improved Fugl-Meyer assessment (FMA) scores, indicating the efficacy of the treatment (Capone et al. 2017). A first-in-human controlled, randomized trial of VNS with upper limb rehabilitation in patients 6 months after ischemic stroke also showed significant improvement in FMA-upper extremity (UE), and Wolf motor function test (WMFT) for the active VNS group. The FMA-UE response was found to go from $33 \%$ in the control group to $88 \%$ in the active group, while WMFT scores increased significantly in day 90 of clinical therapy (Kimberley et al. 2018); this improvement in FMAUE was also seen in patients who underwent VNS paired with upper limb rehabilitation (Dawson et al. 2016). Most recently, VNS was paired with tactile training for treatment of sensory impairment poststroke. Although this "first-in-man" consisted of only one patient, results are promising, with significantly improved sensory function (Kilgard et al. 2018). 
M.J. Capilupi et al.

\section{COAGULOPATHY AND HEMORRHAGIC SHOCK}

Coagulopathy has a significant impact on survival rates for patients involved in trauma, the leading cause of mortality worldwide, because of its role in the development of hemorrhagic shock and other complications (Jacob and $\mathrm{Ku}$ mar 2014). Mortality is as much as four times higher in trauma patients with coagulopathy, and as such, management and treatment strategies are of global health concern. An increase in circulating IL-1 $\beta$ and decreased levels of IL-10 have been shown to be induced by hemorrhage. Rat models have shown that rats that received VNS during resuscitation had IL- $1 \beta$ and IL-10 levels significantly closer to baseline (RezendeNeto et al. 2014). In addition, VNS during resuscitation improved thromboelastomeric parameters, including increased mean clot firmness, increased $\alpha$ angle, and decreased clot-formation time (Rezende-Neto et al. 2014). These improvements in clotting and cytokine levels are likely caused by VNS activating the inflammatory reflex and can potentially lead to enhanced survival in trauma patients. VNS has also been shown to have an effect on the formation of thrombin/antithrombin III (TAT) complexes. In a trial with pigs, VNS significantly increased the level of TAT complexes found in the blood directly shed from the wound. In addition, TAT complex levels were not found to be increased systemically, which suggests that vagus nerve activity modulates thrombin activity directly at the site of injury. Pigs who received VNS therapy were also found to have a significantly shortened period between injury and initiation of clot formation. Finally, animals subjected to VNS were found to have reduced blood flow rates across the wound site (Czura et al. 2010).

Research has also been done to assess the gutprotective effects of VNS in models of hemorrhagic shock and trauma. One study in SpragueDawley rats subjected to trauma/hemorrhagic shock (T/HS), found that VNS significantly decrease gut permeability, lung injury, and neutrophil priming (Levy et al. 2013). These findings indicate that VNS plays a critical role in mediating the production of inflammatory mesenteric lymph in the gut and its circulation system-wide, as well as preventing downstream organ damage after hemorrhagic shock, particularly to the lungs (Levy et al. 2013). Expanding upon these findings, the specific role of CD103 dendritic cells (DCs) in mesenteric lymph after hemorrhagic shock in Sprague-Dawley rats was examined. Rats subjected to T/HS had significantly reduced levels of CD103 and MHC-II DC populations in the mesenteric lymph84, as well as reduced Treg expression in the mesenteric lymph node (Morishita et al. 2014), all of which have previously been shown to play an important role in intestinal immune regulation (Kondělková et al. 2010; Ruane and Lavelle 2011). Animals treated with cervical VNS showed prevention of CD103 and MHC-II reduction in the DC populations in mesenteric lymph, as well as a restoration of Treg expression in the mesenteric lymph node (Morishita et al. 2014).

In addition to traditional methods of VNS treatment, pharmacologic VNS has become an active area of study. Results similar to using traditional VNS were found in a study of male Sprague-Dawley rats, in which injection of T/HS lymph induced a systemic inflammatory response, increased lung permeability, increased recruitment of inflammatory cells to the lungs, and induced acute lung injury (ALI) (Langness et al. 2016). Administration of CPSI-121, a vagal agonist, attenuated these effects, with a significant reduction in levels of macrophage and neutrophils in the lungs; ALI and lung permeability were also mediated by the pharmacologic administration of CPSI-121 (Langness et al. 2016). Similarly, Langness et al. (2016) confirmed that administration of CPSI-121 in Sprague-Dawley rats increased efferent vagus nerve activity, as well as significantly mediated gut and lung injury in T/HS animals (Morishita et al. 2015), making CPSI-121 a potential therapeutic target for future studies in coagulopathy and hemorrhagic shock.

\section{HYPERTENSION}

Hypertension is a prominent healthcare issue in the United States, with nearly 75 million people affected. Hypertension is also a leading risk fac- 
tor in the development of other CVDs, such as heart disease and stroke. Of this population, $\sim 15 \%-18 \%$ have resistant hypertension, making treatment and blood pressure control challenging with available biologics. As such, VNS has been investigated as an alternative, although this field of research is in its infancy. Preliminary results have indicated a promising future in the treatment of hypertension.

Early studies on VNS in induced hypertension in salt-sensitive rats found that 6 weeks of a high-salt diet significantly increased mean arterial pressure, pulse pressure, and number of arrhythmia episodes (Annoni et al. 2015). After 4 weeks of VNS therapy, there was significant improvement seen in both mean arterial pressure and number of arrhythmic episodes in VNS rats compared to controls. These improvements were attributed to changes in the electrophysiology of the heart, including reduction in APD during rapid pacing, slope of APD restitution, special dispersion of action potential, and increase in conduction velocity (Annoni et al. 2015). Similar results have been seen with the use of cardiac-cycle-synchronized selective VNS (ccsVNS) in hypertensive rats in which both static and pulsatile stimulation showed a significant, sustained reduction in hypertension, with static stimulation clinically more effective (Plachta et al. 2016). However, pulsatile stimulation was shown to have a significantly reduced impact on bradycardia, making it more optimal as a treatment strategy (Plachta et al. 2016). Researchers have found similar effects in pigs, in which closed-loop left VNS (CL-LVNS) caused a blood pressure and heart rate decrease in all pigs stimulated through at least two electrode channels (Sevcencu et al. 2018).

Several studies have also been undertaken to assess the effect of VNS when used in conjunction with medications as part of combination therapy, mainly to assess safety, but also to begin to examine its efficacy (Gierthmuehlen and Plachta 2016; Gierthmuehlen et al. 2016a,b). Studies to date have combined selective-afferent VNS (sVNS) with $\alpha 2$-agonists (clonidine), $\beta 1$ selective blockers (metoprolol), and converting enzyme inhibitors (Enalapril). Across studies, the introduction of a biologic was shown to mit- igate the effects of sVNS on blood pressure; however, the treatment was still effective, without inducing significant bradycardia (Gierthmuehlen and Plachta 2016; Gierthmuehlen et al. 2016a,b).

\section{SIDE EFFECTS OF VNS}

While VNS treatment has been shown to produce clinically significant improvement in several cardiovascular conditions, some studies have also shown negative side effects associated with VNS. One of the major side effects of VNS is bradycardia resulting in asystole, a life-threatening condition in which there is a complete cessation of electrical activity from the heart (BenMenachem 2001; Shankar et al. 2013; Pascual 2015). This has only been reported thus far intraoperatively, although case reports have been published citing that VNS was responsible for asystole in patients undergoing long-term VNS for epilepsy (Shankar et al. 2013; Pascual 2015). A case report was published detailing a 55-yearold patient with partial seizures who presented with what appeared to be an increase in atomic seizures on a daily basis $\sim 8$ years following VNS device implantation. While wearing a 24 -h cardiac monitor, he showed recurrent episodes of ventricular standstills, pauses, and runs of complete heart block, as such, increased cardiac monitoring of epilepsy patients who are receiving VNS therapy is recommended (Shankar et al. 2013).

In 2014, another case report was published detailing a patient who experienced late-onset bradycardia and asystole while undergoing VNS treatment for partial epilepsy. The patient experienced recurrent syncope, lightheadedness, difficulty breathing, and loss of consciousness lasting up to $10 \mathrm{sec} \sim 1$ year after implantation. When hospitalized, the patient was found to have sinus bradycardia and a heart rate in the low 30s, which immediately resolved when VNS was deactivated (Pascual 2015). While VNS was likely the cause of these patients' symptoms, the ultimate cause remains unclear.

In addition, infection is a possibility from the surgical implantation of VNS devices. One study indicated that postoperative infections oc- 
M.J. Capilupi et al.

cur in $3 \%$ to $6 \%$ of patients, while another states that infection led to device removal in three of 198 patients (Ben-Menachem 2001). Left cord paralysis is also a possibility during VNS. Symptoms of left cord paralysis, such as pain and hoarseness, typically resolve following the removal of the VNS device (Ben-Menachem 2001). Lower facial weakness has also been seen in patients with implanted VNS devices. While the facial weakness may be attributable to high surgical incisions, the cause is unclear. While deaths have been theorized to be linked to VNS, Ben-Menachem (2001) found that death rates as a result of sudden unexpected, unexplained deaths in epilepsy showed a tendency to be lower in patients receiving VNS.

\section{CONCLUSION}

In conclusion, VNS appears to have a promising future in the treatment of several cardiovascular conditions. It has been shown to aid in the resuscitation of animals in cardiac arrest and with severe hemorrhage, improve recovery after MI, alleviate symptoms of heart failure and stroke, and have the ability to modulate arrhythmias. While these preclinical studies are promising, we must add the cautionary note that serious cardiovascular adverse effects are also quite possible from VNS, like bradycardia and even cardiac standstill. These adverse effects seem to occur when the VNS therapy was used improperly, at a higher than recommend dosage, so caution must be exercised as we progress into human trials of VNS. We look forward to clinical trials evaluating the efficacy of VNS in the treatment of humans with several CVDs in the near future.

\section{REFERENCES}

Al-Khatib SM, Stevenson WG. 2018. Management of ventricular arrhythmias and sudden cardiac death risk related to ischemic and nonischemic cardiomyopathy. JAMA Cardiol 3: 541-542. doi:10.1001/jamacardio.2018.0016

Annoni EM, Xie X, Lee SW, Libbus I, KenKnight BH, Osborn JW, Tolkacheva EG. 2015. Intermittent electrical stimulation of the right cervical vagus nerve in salt-sensitive hypertensive rats: Effects on blood pressure, arrhythmias, and ventricular electrophysiology. Physiol Rep 3: e12476. doi:10.14814/phy2.12476
Ay I, Sorensen AG, Ay H. 2011. Vagus nerve stimulation reduces infarct size in rat focal cerebral ischemia: An unlikely role for cerebral blood flow. Brain Res 1392: 110-115. doi:10.1016/j.brainres.2011.03.060

Bailey P, Bremer F. 1938. A sensory cortical representation of the vagus nerve: with a note on the effects of low blood pressure on the cortical electrogram. J Neurophysiol 1: 405-412. doi:10.1152/jn.1938.1.5.405

Beaumont E, Southerland EM, Hardwick JC, Wright GL, Ryan S, Li Y, KenKnight BH, Armour JA, Ardell JL. 2015. Vagus nerve stimulation mitigates intrinsic cardiac neuronal and adverse myocyte remodeling postmyocardial infarction. Am J Physiol Heart Circ Physiol 309: H1198-H1206. doi:10.1152/ajpheart.00393.2015

Benjamin EJ, Blaha MJ, Chiuve SE, Cushman M, Das SR, Deo R, de Ferranti SD, Floyd J, Fornage M, Gillespie C, et al. 2017. Heart disease and stroke statistics-2017 update. Circulation 135: e146-e603. doi:10.1161/CIR.000000000 0000485

Benjamin EJ, Virani SS, Callaway CW, Chamberlain AM, Chang AR, Cheng S, Chiuve SE, Cushman M, Delling FN, Deo R, et al. 2018. Heart disease and stroke statistics-2018 update: A report from the American Heart Association. Circulation 137: e67-e492. doi:10.1161/CIR .0000000000000558

Ben-Menachem E. 2001. Vagus nerve stimulation, side effects, and long-term safety. J Clin Neurophysiol 18: 415418. doi:10.1097/00004691-200109000-00005

Ben-Menachem E, Mañon-Espaillat R, Ristanovic R, Wilder BJ, Stefan H, Mirza W, Tarver WB, Wernicke JF. 1994. Vagus nerve stimulation for treatment of partial seizures. 1: A controlled study of effect on seizures. First International Vagus Nerve Stimulation Study Group. Epilepsia 35: 616-626. doi:10.1111/j.1528-1157.1994.tb02482.x

Bonaz BL, Bernstein CN. 2013. Brain-gut interactions in inflammatory bowel disease. Gastroenterology 144: 3649. doi:10.1053/j.gastro.2012.10.003

Bonaz B, Sinniger V, Hoffmann D, Clarençon D, Mathieu N, Dantzer C, Vercueil L, Picq C, Trocmé C, Faure P, et al. 2016a. Chronic vagus nerve stimulation in Crohn's disease: A 6-month follow-up pilot study. Neurogastroenterol Motil 28: 948-953. doi:10.1111/nmo.12792

Bonaz B, Sinniger V, Pellissier S. 2016b. Anti-inflammatory properties of the vagus nerve: Potential therapeutic implications of vagus nerve stimulation. J Physiol 594: 57815790. doi:10.1113/JP271539

Borovikova LV, Ivanova S, Zhang M, Yang H, Botchkina GI, Watkins LR, Wang H, Abumrad N, Eaton JW, Tracey KJ. 2000. Vagus nerve stimulation attenuates the systemic inflammatory response to endotoxin. Nature 405: 458462. doi:10.1038/35013070

Brack KE, Patel VH, Coote JH, Ng GA. 2007. Nitric oxide mediates the vagal protective effect on ventricular fibrillation via effects on action potential duration restitution in the rabbit heart. J Physiol 583: 695-704. doi:10.1113/ jphysiol.2007.138461

Cai PY, Bodhit A, Derequito R, Ansari S, Abukhalil F, Thenkabail S, Ganji S, Saravanapavan P, Shekar CC, Bidari S, et al. 2014. Vagus nerve stimulation in ischemic stroke: Old wine in a new bottle. Front Neurol 5: 107. doi:10.3389/ fneur.2014.00107 
Camm F, Luscher T, Maurer G, Serruys P. 2018. The ESC textbook of cardiovascular medicine. Oxford University Press, Oxford.

Capone F, Miccinilli S, Pellegrino G, Zollo L, Simonetti D, Bressi F, Florio L, Ranieri F, Falato E, Di Santo A, et al. 2017. Transcutaneous vagus nerve stimulation combined with robotic rehabilitation improves upper limb function after stroke. Neural Plast 2017: 7876507. doi:10.1155/ 2017/7876507

Carpenter A, Frontera A, Bond R, Duncan E, Thomas G. 2015. Vagal atrial fibrillation: What is it and should we treat it? Int J Cardiol 201: 415-421. doi:10.1016/j.ijcard 2015.08.108

Chen PS, Tan AY. 2007. Autonomic nerve activity and atrial fibrillation. Heart Rhythm 4: S61-S64. doi:10.1016/j hrthm.2006.12.006

Chen M, Yu L, Liu Q, Wang Z, Wang S, Jiang H, Zhou S. 2015. Low level tragus nerve stimulation is a non-invasive approach for anti-atrial fibrillation via preventing the loss of connexins. Int J Cardiol 179: 144-145. doi:10.1016/j ijcard.2014.10.114

Coote JH. 2013. Myths and realities of the cardiac vagus. $J$ Physiol 591: 4073-4085.

Czura CJ, Schultz A, Kaipel M, Khadem A, Huston JM, Pavlov VA, Redl H, Tracey KJ. 2010. Vagus nerve stimulation regulates hemostasis in swine. Shock 33: 608-613. doi:10.1097/SHK.0b013e3181cc0183

Dawson J, Pierce D, Dixit A, Kimberley TJ, Robertson M, Tarver B, Hilmi O, McLean J, Forbes K, Kilgard MP, et al. 2016. Safety, feasibility, and efficacy of vagus nerve stimulation paired with upper-limb rehabilitation after ischemic stroke. Stroke 47: 143-150. doi:10.1161/STROKE AHA.115.010477

Dell P, Olson R. 1951. Thalamic, cortical and cerebellar projections of vagal visceral afferences. $C R$ Seances Soc Biol Fil 145: 1084-1088.

Ferris EB, Capps RB, Weiss S. 1935. Carotid sinus syncope and its bearing on the mechanism of the unconscious state and convulsions. Medicine 14: 377-456.

Gage BF, Waterman AD, Shannon W, Boechler M, Rich MW, Radford MJ. 2001. Validation of clinical classification schemes for predicting stroke: results from the $\mathrm{Na}-$ tional Registry of Atrial Fibrillation. JAMA 285: 28642870. doi:10.1001/jama.285.22.2864

Gierthmuehlen M, Plachta DTT. 2016. Effect of selective vagal nerve stimulation on blood pressure, heart rate and respiratory rate in rats under metoprolol medication. Hypertens Res 39: 79-87. doi:10.1038/hr.2015.122

Gierthmuehlen M, Aguirre D, Cota O, Zentner J, Stieglitz T, Plachta DTT. 2016a. Influence of clonidine on antihypertensive selective afferent vagal nerve stimulation in rats. Neuromodulation 19: 597-606. doi:10.1111/ner.12463

Gierthmuehlen M, Stieglitz T, Zentner J, Plachta DTT. 2016b. Haemodynamic responses to selective vagal nerve stimulation under enalapril medication in rats. PLOS ONE 11: e0147045. doi:10.1371/journal.pone.0147045

Gold MR, Van Veldhuisen DJ, Hauptman PJ, Borggrefe M, Kubo SH, Lieberman RA, Milasinovic G, Berman BJ, Djordjevic S, Neelagaru S, et al. 2016. Vagus nerve stimulation for the treatment of heart failure: The INOVATEHF trial. J Am Coll Cardiol 68: 149-158. doi:10.1016/j.jacc 2016.03.525
Hamann JJ, Ruble SB, Stolen C, Wang M, Gupta RC, Rastog S, Sabbah HN. 2013. Vagus nerve stimulation improves left ventricular function in a canine model of chronic heart failure. Eur J Heart Fail 15: 1319-1326. doi:10 .1093/eurjhf/hft118

Han S, Kobayashi K, Joung B, Piccirillo G, Maruyama M, Vinters HV, March K, Lin SF, Shen C, Fishbein MC, et al. 2012. Electroanatomic remodeling of the left stellate ganglion after myocardial infarction. J Am Coll Cardiol 59: 954-961. doi:10.1016/j.jacc.2011.11.030

Hartupee J, Mann DL. 2013. Positioning of inflammatory biomarkers in the heart failure landscape. J Cardiovasc Transl Res 6: 485-492. doi:10.1007/s12265-013-9467-y

Hays SA, Khodaparast N, Hulsey DR, Ruiz A, Sloan AM, Rennaker RL, Kilgard MP. 2014a. Vagus nerve stimulation during rehabilitative training improves functional recovery after intracerebral hemorrhage. Stroke 45: 3097-3100. doi:10.1161/STROKEAHA.114.006654

Hays SA, Khodaparast N, Ruiz A, Sloan AM, Hulsey DR, Rennaker RL, Kilgard MP. 2014b. The timing and amount of vagus nerve stimulation during rehabilitative training affect poststroke recovery of forelimb strength. Neuroreport 25: 676-682. doi:10.1097/WNR.000000000 0000154

Hays SA, Ruiz A, Bethea T, Khodaparast N, Carmel JB, Rennaker RL, Kilgard MP. 2016. Vagus nerve stimulation during rehabilitative training enhances recovery of forelimb function after ischemic stroke in aged rats. Neurobiol Aging 43: 111-118. doi:10.1016/j.neurobiolaging.2016.03 .030

Heeringa J, van der Kuip DAM, Hofman A, Kors JA, van Herpen G, Stricker BHC, Stijnen T, Lip GYH, Witteman JCM. 2006. Prevalence, incidence and lifetime risk of atrial fibrillation: The Rotterdam study. Eur Heart J 27: 949953. doi:10.1093/eurheartj/ehi825

Heneka MT, O'Banion MK. 2007. Inflammatory processes in Alzheimer's disease. J Neuroimmunol 184: 69-91. doi:10.1016/j.jneuroim.2006.11.017

Hulsey DR, Hays SA, Khodaparast N, Ruiz A, Das P, Rennaker RL, Kilgard MP. 2016. Reorganization of motor cortex by vagus nerve stimulation requires cholinergic innervation. Brain Stimul 9: 174-181. doi:10.1016/j.brs 2015.12.007

Jacob M, Kumar P. 2014. The challenge in management of hemorrhagic shock in trauma. Med J Armed Forces India 70: 163-169. doi:10.1016/j.mjafi.2014.03.001

Jiang Z, Zhao Y, Tsai WC, Yuan Y, Chinda K, Tan J, Onkka P, Shen C, Chen LS, Fishbein MC, et al. 2018. Effects of vagal nerve stimulation on ganglionated plexi nerve activity and ventricular rate in ambulatory dogs with persistent atrial fibrillation. JACC Clin Electrophysiol 4: 1106-1114. doi:10.1016/j.jacep.2018.05.003

Khodaparast N, Hays SA, Sloan AM, Hulsey DR, Ruiz A, Pantoja M, Rennaker RL, Kilgard MP. 2013. Vagus nerve stimulation during rehabilitative training improves forelimb strength following ischemic stroke. Neurobiol Dis 60: 80-88. doi:10.1016/j.nbd.2013.08.002

Khodaparast N, Hays SA, Sloan AM, Fayyaz T, Hulsey DR, Rennaker RL, Kilgard MP. 2014. Vagus nerve stimulation delivered during motor rehabilitation improves recovery in a rat model of stroke. Neurorehabil Neural Repair 28: 698-706. doi:10.1177/1545968314521006 
M.J. Capilupi et al.

Khodaparast N, Kilgard MP, Casavant R, Ruiz A, Qureshi I, Ganzer PD, Rennaker RL, Hays SA. 2016. Vagus nerve stimulation during rehabilitative training improves forelimb recovery after chronic ischemic stroke in rats. Neurorehabil Neural Repair 30: 676-684. doi:10.1177/ 1545968315616494

Kilgard MP, Rennaker RL, Alexander J, Dawson J. 2018. Vagus nerve stimulation paired with tactile training improved sensory function in a chronic stroke patient. NeuroRehabilitation 42: 159-165. doi:10.3233/NRE-172273

Kimberley TJ, Pierce D, Prudente CN, Francisco GE, Yozbatiran N, Smith P, Tarver B, Engineer ND, Alexander Dickie D, Kline DK, et al. 2018. Vagus nerve stimulation paired with upper limb rehabilitation after chronic stroke. Stroke 49: 2789-2792. doi:10.1161/STROKEAHA.118 .022279

Kishi T. 2012. Heart failure as an autonomic nervous system dysfunction. J Cardiol 59: 117-122. doi:10.1016/j.jjcc .2011 .12 .006

Koller-Strametz J, Pacher R, Frey B, Kos T, Woloszczuk W, Stanek B. 1998. Circulating tumor necrosis factor- $\alpha$ levels in chronic heart failure: Relation to its soluble receptor II, interleukin-6, and neurohumoral variables. J Heart Lung Transplant 17: 356-362.

Kolman BS, Verrier RL, Lown B. 1975. The effect of vagus nerve stimulation upon vulnerability of the canine ventricle: Role of sympathetic-parasympathetic interactions. Circulation 52: 578-585. doi:10.1161/01.CIR.52.4.578

Kondělková K, Vokurková D, Krejsek J, Borská L, Fiala Z, Ctirad A. 2010. Regulatory T cells (TREG) and their roles in immune system with respect to immunopathological disorders. Acta Medica 53: 73-77.

Kong SS, Liu JJ, Hwang TC, Yu XJ, Zhao M, Zhao M, Yuan BX, Lu Y, Kang YM, Wang B, et al. 2012. Optimizing the parameters of vagus nerve stimulation by uniform design in rats with acute myocardial infarction. PLoS ONE 7: e42799. doi:10.1371/journal.pone.0042799

Koopman FA, Chavan SS, Miliko S, Grazio S, Sokolovic S, Schuurman PR, Mehta AD, Levine YA, Faltys M, Zitnik R, et al. 2016. Vagus nerve stimulation inhibits cytokine production and attenuates disease severity in rheumatoid arthritis. Proc Natl Acad Sci 113: 8284-8289. doi:10.1073/ pnas. 1605635113

Langness S, Costantini TW, Morishita K, Eliceiri BP, Coimbra R. 2016. Modulating the biologic activity of mesenteric lymph after traumatic shock decreases systemic inflammation and end organ injury. PLOS ONE 11: e0168322. doi:10.1371/journal.pone.0168322

Laurita KR, Hirose M. 2013. Electrical vagal stimulation and cardioprotection. Heart Rhythm 10: 1708-1709. doi:10 $.1016 /$ j.hrthm.2013.08.033

Lee SW, Anderson A, Guzman PA, Nakano A, Tolkacheva EG, Wickman K. 2018a. Atrial GIRK channels mediate the effects of vagus nerve stimulation on heart rate dynamics and arrhythmogenesis. Front Physiol 9: 943. doi:10.3389/fphys.2018.00943

Lee SW, Kulkarni K, Annoni EM, Libbus I, KenKnight BH, Tolkacheva EG. 2018b. Stochastic vagus nerve stimulation affects acute heart rate dynamics in rats. PLOS ONE 13: e0194910. doi:10.1371/journal.pone.0194910

Lerner DL, Yamada KA, Schuessler RB, Saffitz JE. 2000. Accelerated onset and increased incidence of ventricular arrhythmias induced by ischemia in $\mathrm{Cx} 43$-deficient mice. Circulation 101: 547-552. doi:10.1161/01.CIR.101.5.547

Levine B, Kalman J, Mayer L, Fillit HM, Packer M. 1990. Elevated circulating levels of tumor necrosis factor in severe chronic heart failure. $N$ Engl J Med 323: 236-241. doi:10.1056/NEJM199007263230405

Levy MN, Blattberg B. 1976. Effect of vagal stimulation on the overflow of norepinephrine into the coronary sinus during cardiac sympathetic nerve stimulation in the dog. Circ Res 38: 81-84. doi:10.1161/01.RES.38.2.81

Levy G, Fishman JE, Xu D, Chandler BTJ, Feketova E, Dong W, Qin Y, Alli V, Ulloa L, Deitch EA. 2013. Parasympathetic stimulation via the vagus nerve prevents systemic organ dysfunction by abrogating gut injury and lymph toxicity in trauma and hemorrhagic shock. Shock 39: 39-44. doi:10.1097/SHK.0b013e31827b450d

Li S, Zhou X, Yu L, Jiang H. 2015. Low level non-invasive vagus nerve stimulation: A novel feasible therapeutic approach for atrial fibrillation. Int J Cardiol 182: 189-190. doi:10.1016/j.ijcard.2014.12.171

Mann DL. 2015. Innate immunity and the failing heart: The cytokine hypothesis revisited. Circ Res 116: 1254-1268. doi:10.1161/CIRCRESAHA.116.302317

Meregnani J, Clarençon D, Vivier M, Peinnequin A, Mouret C, Sinniger V, Picq C, Job A, Canini F, Jacquier-Sarlin M, et al. 2011. Anti-inflammatory effect of vagus nerve stimulation in a rat model of inflammatory bowel disease. Auton Neurosci 160: 82-89. doi:10.1016/j.autneu.2010 .10 .007

Meyers EC, Solorzano BR, James J, Ganzer PD, Lai ES, Rennaker RL, Kilgard MP, Hays SA. 2018. Vagus nerve stimulation enhances stable plasticity and generalization of stroke recovery. Stroke 49: 710-717. doi:10.1161/STRO KEAHA.117.019202

Miller AH, Maletic V, Raison CL. 2009. Inflammation and its discontents: The role of cytokines in the pathophysiology of major depression. Biol Psychiatry 65: 732-741. doi:10 $.1016 /$ j.biopsych.2008.11.029

Morishita K, Costantini TW, Eliceiri B, Bansal V, Coimbra R. 2014. Vagal nerve stimulation modulates the dendritic cell profile in posthemorrhagic shock mesenteric lymph. J Trauma Acute Care Surg 76: 610-617, discussion 617618. doi:10.1097/TA.0000000000000137

Morishita K, Costantini TW, Ueno A, Bansal V, Eliceiri B, Coimbra R. 2015. A pharmacologic approach to vagal nerve stimulation prevents mesenteric lymph toxicity after hemorrhagic shock. J Trauma Acute Care Surg 78: 52-58, discussion 58-59. doi:10.1097/TA.000000000000 0489

Mozaffarian D, Benjamin EJ, Go AS, Arnett DK, Blaha MJ, Cushman M, de Ferranti S, Després JP, Fullerton HJ, Howard VJ, et al. 2015. Heart disease and stroke statistics-2015 update: A report from the American Heart Association. Circulation 131: e29-e322. doi:10.1161/ CIR.0000000000000152

Mravec B. 2010. The role of the vagus nerve in stroke. Auton Neurosci 158: 8-12. doi:10.1016/j.autneu.2010.08.009

Naggar I, Nakase K, Lazar J, Salciccioli L, Selesnick I, Stewart M. 2014. Vagal control of cardiac electrical activity and wall motion during ventricular fibrillation in large animals. Auton Neurosci 183: 12-22. doi:10.1016/j.autneu .2014 .01 .009 
Nearing BD, Libbus I, Amurthur B, Kenknight BH, Verrier RL. 2016. Acute autonomic engagement assessed by heart rate dynamics during vagus nerve stimulation in patients with heart failure in the ANTHEM-HF Trial: Acute heart rate dynamics during VNS. J Cardiovasc Electrophysiol 27: 1072-1077. doi:10.1111/jce.13017

Ng GA. 2014. Vagal modulation of cardiac ventricular arrhythmia. Exp Physiol 99: 295-299. doi:10.1113/expphy siol.2013.072652

Nozaki N, Yamaguchi S, Shirakabe M, Nakamura H, Tomoike H. 1997. Soluble tumor necrosis factor receptors are elevated in relation to severity of congestive heart failure. Jpn Circ J 61: 657-664. doi:10.1253/jcj.61.657

Olofsson PS, Katz DA, Rosas-Ballina M, Levine YA, Ochani M, Valdés-Ferrer SI, Pavlov VA, Tracey KJ, Chavan SS. 2012. $\alpha 7$ nicotinic acetylcholine receptor $(\alpha 7 \mathrm{nAChR})$ expression in bone marrow-derived non- $\mathrm{T}$ cells is required for the inflammatory reflex. Mol Med 18: 539-543. doi:10 .2119/molmed.2011.00405

Ovbiagele B, Goldstein LB, Higashida RT, Howard VJ, Johnston SC, Khavjou OA, Lackland DT, Lichtman JH, Moh S, Sacco RL, et al. 2013. Forecasting the future of stroke in the United States: A policy statement from the American Heart Association and American Stroke Association. Stroke 44: 2361-2375. doi:10.1161/STR.0b013e31829 $734 f 2$

Parrish WR, Rosas-Ballina M, Gallowitsch-Puerta M, Ochani M, Ochani K, Yang LH, Hudson L, Lin X, Patel $\mathrm{N}$, Johnson SM, et al. 2008. Modulation of TNF release by choline requires $\alpha 7$ subunit nicotinic acetylcholine receptor-mediated signaling. Mol Med 14: 567-574. doi:10 .2119/2008-00079.Parrish

Pascual FT. 2015. Vagus nerve stimulation and late-onset bradycardia and asystole: Case report. Seizure 26: 5-6. doi:10.1016/j.seizure.2015.01.006

Pavlov VA, Chavan SS, Tracey KJ. 2018. Molecular and functional neuroscience in immunity. Annu Rev Immunol 36: 783-812. doi:10.1146/annurev-immunol-042617-053158

Penry JK, Dean JC. 1990. Prevention of intractable partial seizures by intermittent vagal stimulation in humans: Preliminary results. Epilepsia 31(Suppl 2): S40-S43.

Plachta DTT, Zentner J, Aguirre D, Cota O, Stieglitz T, Gierthmuehlen M. 2016. Effect of cardiac-cycle-synchronized selective vagal stimulation on heart rate and blood pressure in rats. $A d v$ Ther 33: 1246-1261. doi:10.1007/ s12325-016-0348-Z

Qi B, Wei Y, Chen S, Zhou G, Li H, Xu J, Ding Y, Lu X, Zhao L, Zhang F, et al. 2014. Nav1.8 channels in ganglionated plexi modulate atrial fibrillation inducibility. Cardiovasc Res 102: 480-486. doi:10.1093/cvr/cvu005

Rezende-Neto JB, Alves RL, Carvalho M, Almeida T, Trant C, Kushmerick C, Andrade M, Rizoli SB, Cunha-Melo J. 2014. Vagus nerve stimulation improves coagulopathy in hemorrhagic shock: A thromboelastometric animal model study. J Trauma Manag Outcomes 8: 15. doi:10.1186/ 1752-2897-8-15

Ronson RS, Puskas JD, Thourani VH, Velez DA, Bufkin BL, Glass J, Guyton RA, Vinten-Johansen J. 2003. Controlled intermittent asystole cardiac therapy induced by pharmacologically potentiated vagus nerve stimulation in normal and hibernating myocardium. Ann Thorac Surg 75: 1929-1936. doi:10.1016/S0003-4975(03)00115-2
Rordorf R, Savastano S, Sanzo A, Spazzolini C, De Amici M Camporotondo R, Ghio S, Vicentini A, Petracci B, De Regibus V, et al. 2014. Tumor necrosis factor- $\alpha$ predicts response to cardiac resynchronization therapy in patients with chronic heart failure. Circ J 78: 2232-2239. doi:10 .1253/circj.CJ-14-0023

Ruane DT, Lavelle EC. 2011. The role of $\mathrm{CD}_{103}{ }^{+}$dendritic cells in the intestinal mucosal immune system. Front Immunol 2: 25. doi:10.3389/fimmu.2011.00025

Sabbah HN, Ilsar I, Zaretsky A, Rastogi S, Wang M, Gupta RC. 2011. Vagus nerve stimulation in experimental heart failure. Heart Fail Rev 16: 171-178. doi:10.1007/s10741010-9209-z

Sanchis-Gomar F, Perez-Quilis C, Leischik R, Lucia A. 2016. Epidemiology of coronary heart disease and acute coronary syndrome. Ann Transl Med 4: 256. doi:10.21037/atm .2016 .06 .33

Sarnoff SJ, Gilmore JP, Brockman SK, Mitchell JH, Linden RJ. 1960. Regulation of ventricular contraction by the carotid sinus. Its effect on atrial and ventricular dynamics. Circ Res 8: 1123-1136. doi:10.1161/01.RES.8.5.1123

Savarese G, Lund LH. 2017. Global public health burden of heart failure. Card Fail Rev 3: 7-11. doi:10.15420/cfr.2016 $: 25: 2$

Schacter S, Schmidt D. 2000. Vagus nerve stimulation. CRC, Boca Raton, FL.

Schmitt J, Duray G, Gersh BJ, Hohnloser SH. 2009. Atrial fibrillation in acute myocardial infarction: A systematic review of the incidence, clinical features and prognostic implications. Eur Heart J 30: 1038-1045. doi:10.1093/eu rheartj/ehn579

Sevcencu C, Nielsen TN, Struijk JJ. 2018. An intraneural electrode for bioelectronic medicines for treatment of hypertension. Neuromodulation 21: 777-786. doi:10.1111/ ner. 12758

Shankar R, Olotu VO, Cole N, Sullivan H, Jory C. 2013. Case report: Vagal nerve stimulation and late onset asystole. Seizure 22: 312-314. doi:10.1016/j.seizure.2012.12.011

Sheng X, Scherlag BJ, Yu L, Li S, Ali R, Zhang Y, Fu G, Nakagawa H, Jackman WM, Lazzara R, et al. 2011. Prevention and reversal of atrial fibrillation inducibility and autonomic remodeling by low-level vagosympathetic nerve stimulation. J Am Coll Cardiol 57: 563-571. doi:10.1016/j.jacc.2010.09.034

Shinlapawittayatorn K, Chinda K, Palee S, Surinkaew S, Kumfu S, Kumphune S, Chattipakorn S, Kenknight BH, Chattipakorn N. 2014. Vagus nerve stimulation initiated late during ischemia, but not reperfusion, exerts cardioprotection via amelioration of cardiac mitochondrial dysfunction. Heart Rhythm 11: 2278-2286. doi:10.1016/j .hrthm.2014.08.001

Staerk L, Sherer JA, Ko D, Benjamin EJ, Helm RH. 2017. Atrial fibrillation: Epidemiology, pathophysiology, and clinical outcomes. Circ Res 120: 1501-1517. doi:10 .1161/CIRCRESAHA.117.309732

Sun P, Wang J, Zhao S, Yang Z, Tang Z, Ravindra N, Bradley J, Ornato JP, Peberdy MA, Tang W. 2018. Improved outcomes of cardiopulmonary resuscitation in rats treated with vagus nerve stimulation and its potential mechanism. Shock 49: 698-703. doi:10.1097/SHK.0000000000 000962 
M.J. Capilupi et al.

Tracey KJ. 2002. The inflammatory reflex. Nature 420: $853-$ 859. doi:10.1038/nature01321

Tsutsumi T, Ide T, Yamato M, Kudou W, Andou M, Hirooka Y, Utsumi H, Tsutsui H, Sunagawa K. 2008. Modulation of the myocardial redox state by vagal nerve stimulation after experimental myocardial infarction. Cardiovasc Res 77: 713-721. doi:10.1093/cvr/cvm092

Vaseghi M, Salavatian S, Rajendran PS, Yagishita D, Woodward WR, Hamon D, Yamakawa K, Irie T, Habecker BA Shivkumar K. 2017. Parasympathetic dysfunction and antiarrhythmic effect of vagal nerve stimulation following myocardial infarction. JCI Insight 2: 86715. doi:10.1172/ jci.insight. 86715

Wang Z, Zhou X, Sheng X, Yu L, Jiang H. 2015. Unilateral low-level transcutaneous electrical vagus nerve stimulation: A novel noninvasive treatment for myocardial infarction. Int J Cardiol 190: 9-10. doi:10.1016/j.ijcard.2015 .04 .087

Weisfeldt ML, Becker LB. 2002. Resuscitation after cardiac arrest: A 3-phase time-sensitive model. JAMA 288: 30353038. doi:10.1001/jama.288.23.3035

Winter J, Tipton MJ, Shattock MJ. 2018. Autonomic conflict exacerbates long QT associated ventricular arrhythmias. $J$ Mol Cell Cardiol 116: 145-154. doi:10.1016/j.yjmcc.2018 .02 .001

Wolf PA, Abbott RD, Kannel WB. 1991. Atrial fibrillation as an independent risk factor for stroke: The Framingham Study. Stroke 22: 983-988. doi:10.1161/01.STR.22.8.983

Xie X, Lee SW, Johnson C, Ippolito J, KenKnight BH, Tolkacheva EG. 2014. Intermittent vagal nerve stimulation alters the electrophysiological properties of atrium in the myocardial infarction rat model. Conf Proc IEEE Eng Med Biol Soc 2014: 1575-1578. doi:10.1109/EMBC .2014 .6943904

Yu L, Scherlag BJ, Sha Y, Li S, Sharma T, Nakagawa H, Jackman WM, Lazzara R, Jiang H, Po SS. 2012. Interactions between atrial electrical remodeling and autonomic remodeling: How to break the vicious cycle. Heart Rhythm 9: 804-809. doi:10.1016/j.hrthm.2011.12.023

Yuan H, Silberstein SD. 2016a. Vagus nerve and vagus nerve stimulation, a comprehensive review: Part II. Headache 56: 259-266. doi:10.1111/head.12650

Yuan H, Silberstein SD. 2016b. Vagus nerve and vagus nerve stimulation, a comprehensive review: Part III. Headache 56: 479-490. doi:10.1111/head.12649

Zabara J. 1985. Peripheral control of hypersynchronous discharge in epilepsy. Electroencephal Clin Neurophysiol 61: S162. doi:10.1016/0013-4694(85)90626-1

Zannad F, De Ferrari GM, Tuinenburg AE, Wright D, Brugada J, Butter C, Klein H, Stolen C, Meyer S, Stein KM, et al. 2015. Chronic vagal stimulation for the treatment of low ejection fraction heart failure: Results of the NEural Cardiac TherApy foR Heart Failure (NECTAR-HF) randomized controlled trial. Eur Heart J 36: 425-433. doi:10 .1093/eurheartj/ehu345

Zhang Y, Chen A, Song L, Li M, Luo Z, Zhang W, Chen Y, He B. 2016. Low-level vagus nerve stimulation reverses cardiac dysfunction and subcellular calcium handling in rats with post-myocardial infarction heart failure. Int Heart $J$ 57: 350-355. doi:10.1536/ihj.15-516 


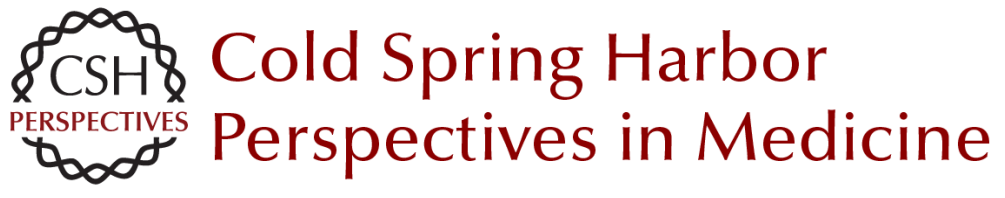

\title{
Vagus Nerve Stimulation and the Cardiovascular System
}

\author{
Michael J. Capilupi, Samantha M. Kerath and Lance B. Becker \\ Cold Spring Harb Perspect Med 2020; doi: 10.1101/cshperspect.a034173 originally published online May \\ 20, 2019
}

\section{Subject Collection Bioelectronic Medicine}

Neural Control of Inflammation: Bioelectronic Medicine in Treatment of Chronic Inflammatory

Disease

Michael Eberhardson, Laura Tarnawski, Monica

Centa, et al.

Noninvasive Neuromodulation of Peripheral Nerve

Pathways Using Ultrasound and Its Current

Therapeutic Implications

Christopher Puleo and Victoria Cotero

Enteric Neuromodulation for the Gut and Beyond

Yogi A. Patel and Pankaj J. Pasricha

\section{Optogenetic Control of the Peripheral Nervous System}

Rui B. Chang

Closed-Loop Neuromodulation in Physiological and Translational Research Stavros Zanos

Electrical Impedance Methods in Neuromuscular Assessment: An Overview

Seward B. Rutkove and Benjamin Sanchez

Optogenetic Medicine: Synthetic Therapeutic

Solutions Precision-Guided by Light Haifeng Ye and Martin Fussenegger

Technobiology's Enabler: The Magnetoelectric Nanoparticle Sakhrat Khizroev
Bioelectronic Medicine: From Preclinical Studies on the Inflammatory Reflex to New Approaches in Disease Diagnosis and Treatment

Valentin A. Pavlov, Sangeeta S. Chavan and Kevin J. Tracey

Vagus Nerve Stimulation and the Cardiovascular System Michael J. Capilupi, Samantha M. Kerath and Lance B. Becker

Harnessing the Inflammatory Reflex for the Treatment of Inflammation-Mediated Diseases Yaakov A. Levine, Michael Faltys and David Chernoff

Recording and Decoding of Vagal Neural Signals Related to Changes in Physiological Parameters and Biomarkers of Disease

Theodoros P. Zanos

Restoring Movement in Paralysis with a

Bioelectronic Neural Bypass Approach: Current

State and Future Directions Chad E. Bouton

Bioelectronic Medicine--Ethical Concerns Samuel Packer, Nicholas Mercado and Anita Haridat

Use of Bioelectronics in the Gastrointestinal Tract Larry Miller, Aydin Farajidavar and Anil Vegesna

Vagus Nerve Stimulation at the Interface of BrainGut Interactions

Bruno Bonaz, Valérie Sinniger and Sonia Pellissier

For additional articles in this collection, see http://perspectivesinmedicine.cshlp.org/cgi/collection/ 